\title{
Tomodensitometric Aspects of Acute Intestinal Ischemia: A Prospective Study of 20 Cases
}

\author{
Yomboe Abel Bamouni1,2, Adjirata Koama ${ }^{*}$ (1) , Bénilde Marie Ange Tiemtore-Kambou2,3, \\ Lobna Ben Temellist ${ }^{4}$, Nina Astrid N'de-Ouédraogo ${ }^{2,3}$, Massara Koné Sigué1, \\ Habiba Mizouni ${ }^{4}$, Ousséni Diallo ${ }^{1,2}$, Rabiou Cissé ${ }^{1,2}$ \\ ${ }^{1}$ YALGADO OUEDRAOGO University Hospital Centre (CHUYO), Ouagadougou, Burkina Faso \\ ${ }^{2}$ Professor JOSEPH KI ZERBO Ouaga I University, Ouagadougou, Burkina Faso \\ ${ }^{3}$ University Hospital Centre of BOGODOGO (CHUB), Ouagadougou, Burkina Faso \\ ${ }^{4}$ University Hospital Centre la RABTA of Tunis, Tunis, Tunisia \\ Email: ^adjikoama@gmail.com, abelbamouni@hotmail.com, kbenildema@yahoo.fr,drlbt@hotmail.com, ninawed@hotmail.com, \\ massarakone56@gmail.com, habiba.mizouni@gmail.com,odiallo75@yahoo.fr, ciss.rabi@gmail.com
}

How to cite this paper: Bamouni, Y.A., Koama, A., Tiemtore-Kambou, B.M.A., Temellist, L.B., N'de-Ouédraogo, N.A., Sigué, M.K., Mizouni, H., Diallo, O. and Cissé, R. (2019) Tomodensitometric Aspects of Acute Intestinal Ischemia: A Prospective Study of 20 Cases. Open Journal of Radiology, 9, 48-57. https://doi.org/10.4236/ojrad.2019.91005

Received: January 3, 2019

Accepted: January 28, 2019

Published: February 1, 2019

Copyright () 2019 by author(s) and Scientific Research Publishing Inc. This work is licensed under the Creative Commons Attribution International License (CC BY 4.0).

http://creativecommons.org/licenses/by/4.0/ (c) (i) Open Access

\begin{abstract}
Background: Acute intestinal ischemia is an abdominal suffering occasioned by a sudden reduction of mesenteric blood circulation. It is a rare and potentially serious medical-surgical emergency, deadly in $50 \%$ to $100 \%$ of cases. Diagnosis is known to be difficult at the early stage during which a well-conducted treatment may help reduce mortality. Multi-detector scanners were assessed to be far more sensitive and appropriate for the diagnosis of mesenteric ischemia than angiography. Objective: Study the tomodensitometric aspects of acute intestinal ischemia, and the traps of late diagnosis, in order to optimize the care and improve the prognosis of this disease. Patients and Methods: This is a prospective, longitudinal and descriptive study covering a period of nine (9) months. We have included all cases of acute intestinal ischemia. Results: Twenty (20) cases of acute intestinal ischemia were registered i.e. a frequency of 2.2 cases per month. The average age of the patients was 67.8 years with 0.7 as sex ratio. Abdominal pain was noted in all cases. History of cardiovascular disease was found in seven (7) cases. Abdominal angioscan was performed in fifteen (15) cases. There was a suspected diagnosis upon request in ten (10) cases. The main intestinal lesions were the lack of parietal enhancement (13 cases) and parietal thickening (12 cases). There were sixteen (16) cases of acute mesenteric ischemia and four (4) cases of ischemic colitis. The acute mesenteric ischemia was arterial in twelve (12) cases, venous in two (2) cases and mixed in two (2) cases. Diagnosis was made at the stage of intestinal infarction in eleven (11) cases. Ten (10) patients were operated and 10 others were treated medically. Thirteen (13) cases of death were registered. Conclusion: Acute intestinal ischemia is a rare but serious disease. Abdominal pain
\end{abstract}


is the main ground for consultation. Diagnosis is often late and focuses on abdominal CT angiography. An early diagnosis could help improve its prognosis.

\section{Keywords}

Acute Intestinal Ischemia, Mesenteric Infarction, Angioscan, Abdominal Pain

\section{Introduction}

Acute intestinal ischemia is an abdominal suffering occasioned by a sudden reduction of mesenteric blood circulation [1]. It can be organic or functional, permanent or temporary [2]. The concerned digestive structures include those that are vascularized by the celiac trunk, the upper and lower mesenteric arteries, as well as the portal vein pattern (portal vein, upper and lower mesenteric arteries and the splenic vein) [3]. Classically, two entities are described: the arterial, venous or low flow mesenteric ischemia and ischemic colitis [3]. It is a rare and potentially serious medical-surgical emergency, occasioning death in $50 \%$ to $100 \%$ of cases [1] [2] [3]. Diagnosis is known to be difficult at the early stage during which a well-conducted treatment may help reduce mortality [3]. Multi-detector scanners were assessed to be far more sensitive and appropriate in the diagnosis of mesenteric ischemia than angiography [4]. We have therefore undertaken to highlight through the cases diagnosed at the RABTA Hospital of Tunis, the tomodensitometric aspects of acute intestinal ischemia and the traps of late diagnosis, in order to optimize the care and improve the prognosis of this disease.

\section{Patients and Methods}

Type and period of study: This is a prospective, longitudinal and descriptive study covering a period of nine (9) months from $1^{\text {st }}$ February to $30^{\text {th }}$ October, 2016.

Site of study: The Radiology, Medical-Surgical Emergency, General Surgery, Cardiovascular Surgery and Gastro-Enterology Units of the RABTA hospital in Tunis, served as the framework of study. The angioscan was available and regularly used in the Radiology Unit.

Population of study: We included all patients admitted to the Radiology Unit and the abdominal scan revealed signs of acute intestinal ischemia.

Data collection and analysis: The data collection was done prospectively with a previously established data sheet. Anytime lesions of acute intestinal ischemia were noted in a patient, we went to his unit of origin to collect the information relating to his history, functional signs, scanner decisions and evolution. The data were typed and analyzed with a micro-computer using the Excel and Word softwares 2010 versions.

Scanner device: The scanning examinations were done with a multi-detector Siemens Somatom plus 128 baretts scanner which is functional since 2016. 
Ethical aspects: We got the authorization from the Hospital's director, as well as all the various heads of the units concerned. We also got the informed consent of the patients or their dependents. Anonymity was respected for all the patients.

\section{Results}

\subsection{Sociodemographic and Clinical-Biological Data}

Within nine months, we registered 20 cases of acute intestinal ischemia, i.e. 2.2 cases per month. There were 12 women and 8 men. The patients' average age was 67.8 years with extremes of 43 and 96 years. Cardiovascular history (high blood pressure, atrial fibrillation cardiac arytmy, atherosclerosis, coronary failure) was noted in 07 cases. Chronic renal failure was found in 05 cases. Five (05) patients did not have any known disease history. The diagnosis of acute intestinal ischemia was suspected by the clinician in 10 cases over the 20. A generalized acute peritonitis was suspected in 04 patients.

\subsection{Scanner Data}

The average deadline for the scan after the outbreak of the symptoms was 54 hours i.e. 2.25 days with extremes of 1 and 15 days.

\subsubsection{Technical Data}

Technically speaking, an abdominal angioscan was performed in 15 cases and an abdominal scan with injection of a contrast dye in portal phase in 05 cases. In all cases where intestinal ischemia was suspected, we performed an abdominal angioscan without injection, and then with injection of contrast dye in the arterial and portal phases.

\subsubsection{Semiological Data}

Figure 1 represents the distribution of intestinal lesions: The lack of parietal heightening was the most frequent lesion, noted in 13 cases (Figure 2(A)), followed by parietal thickening noted in 12 cases (Figure 2(A)).

Serious symptoms are represented in Table 1: Sixteen patients were having serious symptoms. There was intraabdominal fluid effusion in 13 cases, parietal pneumatosis in 06 (Figure 2(B)), aeromesentery in 02 cases (Figure 2(C)), hepatic portal venous gas in 01 case, parietal tightening in 02 cases (Figure $2(\mathrm{C})$ ), pneumoperitoneum in one case and intestinal distention in 03 cases (Figure 2(C)).

The vascular lesions are distributed in Table 2: We noted 16 cases of mesenteric ischemia and 04 cases of ischemic colitis. The mesenteric ischemia was arterial in $75 \%$, venous in $12 \%$ and mixed in $12 \%$ of cases. Partial or total occlusion of the upper mesenteric artery (UMA) was noted in 10 cases (Figure 3(A)-(C)). Occlusion of the portal vein was noted in 03 cases (Figure 3(D)) and upper mesenteric vein (UMV) occlusion in 03 cases (Figure 3(C) and Figure 3(D)). In 07 cases, no vascular occlusion was viewed. Diffuse atherosclerosis was revealed in 09 cases (Figure 3(B)) and 01 case of left intraventricular embolism was found.

Table 3 illustrates the associated lesions: Visceral infarction (Figure 2(D)) 


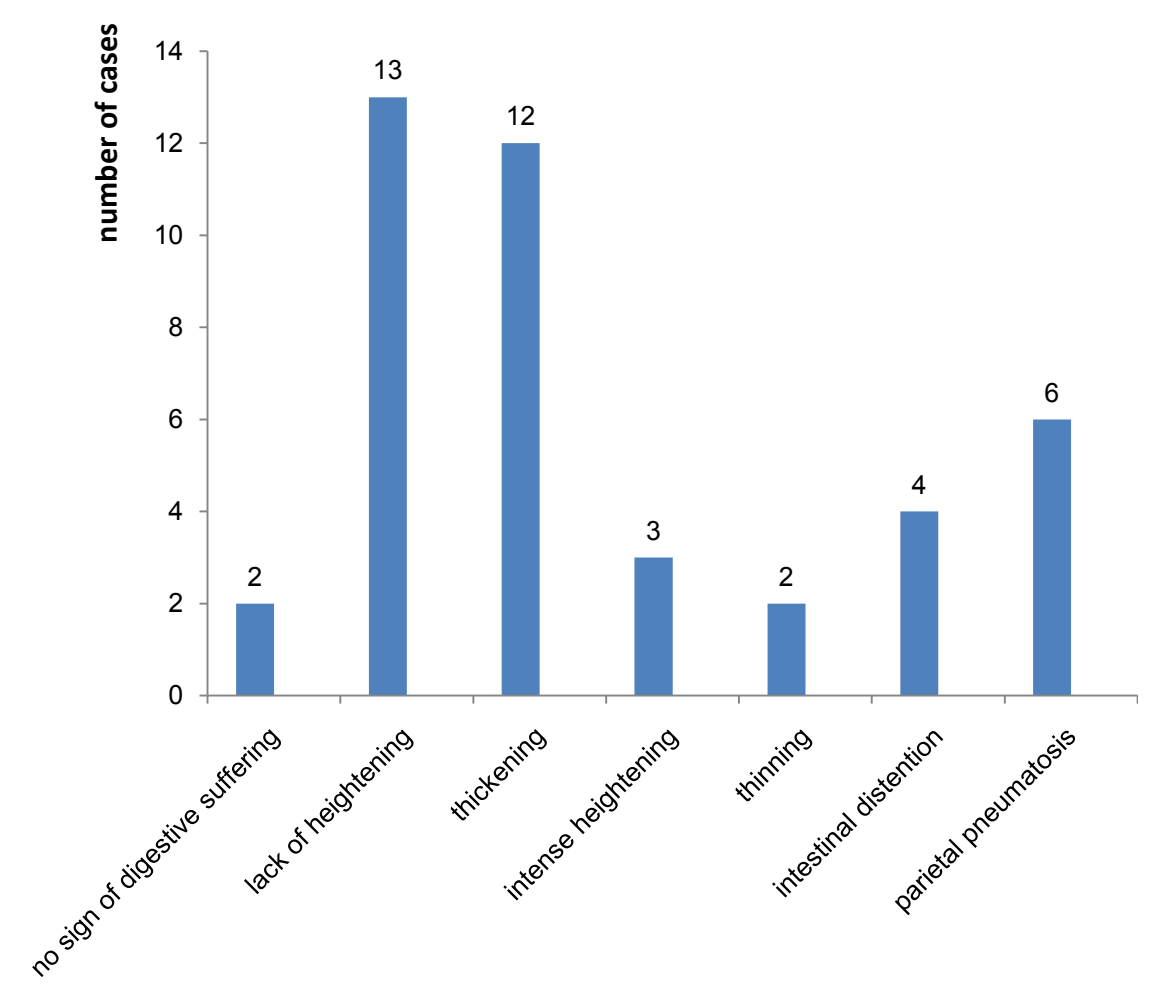

Figure 1. Distribution of patients according to the intestinal lesions. $\mathrm{n}=20$.
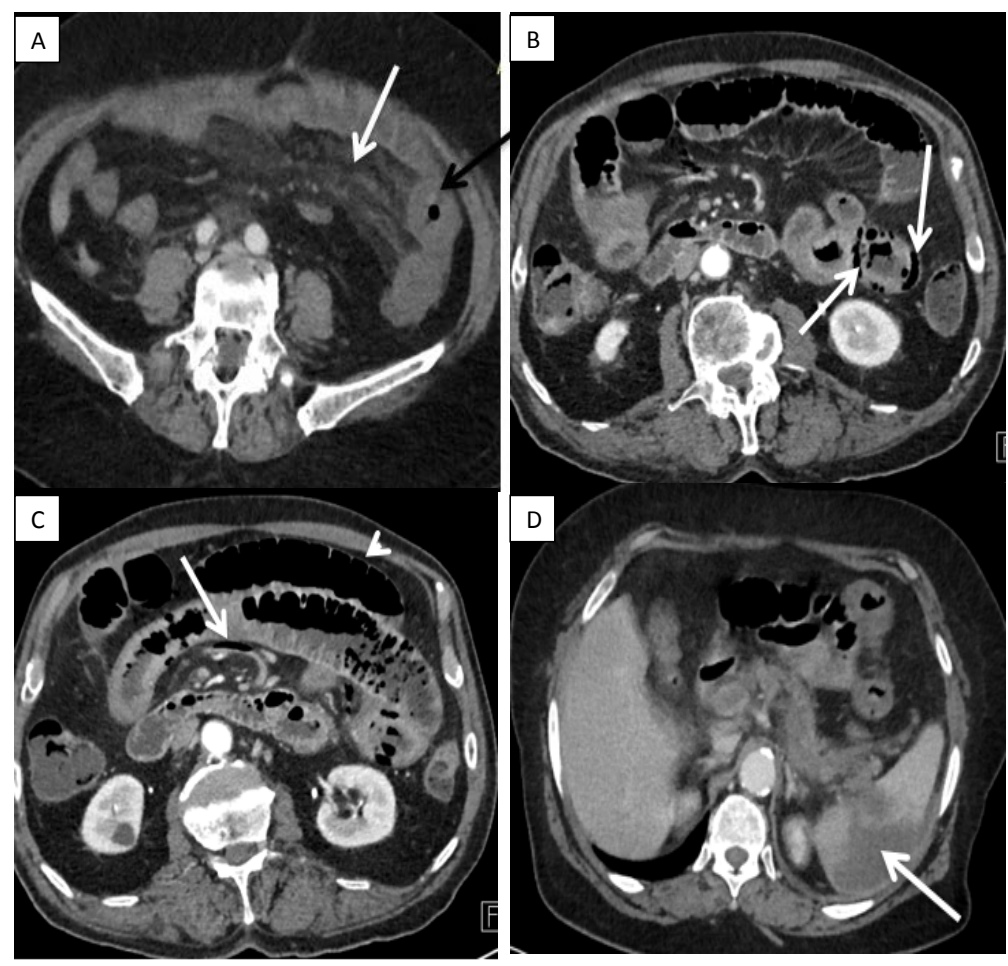

Figure 2. Abdominal angioscan with injection of contrast dye in portal phases, axial sections. (A) Thickening with lack of heightening of the left colonic lining (black arrow); mesenteric infiltration (white arrow); (B) Aeromesentery (white arrow), parietal tightening with intestinal distention (head of white arrow); (C) Parietalpneumatosis (white arrows); (D) Splenic infarction (white arrows). 


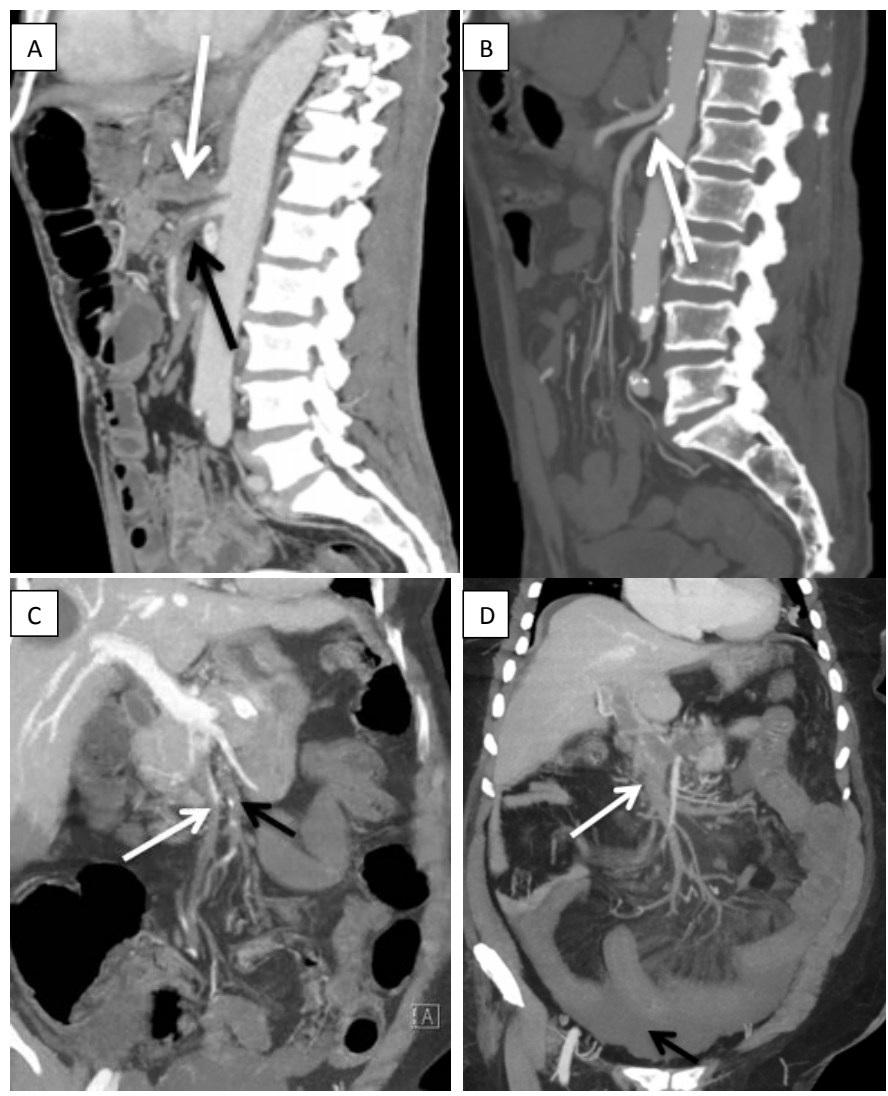

Figure 3. Abdominal angioscan with injection of contrast dye, in arterial phase in sagittal MIP reconstruction (A \& B); coronal MIP reconstitution in portal phase (C \& D). (A) Total obstruction of the celiac trunk (white arrow), total obstruction of the upper mesenteric artery (black arrow); (B) Stenosis of the upper mesenteric artery at $70 \%$ in its beginning by a plaque of calcified atheromas (white arrow), diffuse atheromatous infiltration of the abdominal aorta; (C) Mixed obstruction of the upper mesenteric artery (black arrow) and the upper mesenteric vein (white arrow); (D) Total obstruction of the mesenteric-portal venous axis (white arrow), intraabdominal fluid effusion (black arrow).

Table 1. Distribution of patients according to symptoms of seriousness $(n=16)$.

\begin{tabular}{ccc}
\hline Symptoms of seriousness & Number of cases & Percentages \\
\hline Intra-abdominalfluid & 13 & $80 \%$ \\
Parietalpneumatosis pariétale & 06 & $37 \%$ \\
Aeromesentery & 02 & $12.5 \%$ \\
Hepatic portal venousgas & 01 & $6.25 \%$ \\
Parietaltightening & 02 & $12.5 \%$ \\
Pneumoperitoneum & 01 & $6.25 \%$ \\
Intestinal distention & 03 & $18.75 \%$ \\
\hline
\end{tabular}

Table 2. Distribution of patients according to the vascular lesions $(n=20)$.

\begin{tabular}{ccc}
\hline Vasculaires lesions & Number of cases & Percentages \\
\hline Lack of vascular occlusion: & 07 & $35 \%$ \\
\hline
\end{tabular}




\section{Continued}

\begin{tabular}{ccc}
\hline Occlusion of the UMA & 10 & $50 \%$ \\
Occlusion of the celiac trunk & 01 & $5 \%$ \\
Occlusion of the LMA & 01 & $5 \%$ \\
Occlusion of the PT & 03 & $15 \%$ \\
Occlusion of theUMV & 03 & $15 \%$ \\
Occlusion of the LMV & 02 & $10 \%$ \\
Occlusion of the splenic vein & 02 & $10 \%$ \\
Atherosclerosis & 09 & $45 \%$ \\
Intraauricular thrombus & 01 & $5 \%$
\end{tabular}

UMA: upper mesenteric artery; LMA: lower mesenteric artery; PT: portal trunk; UMV: upper mesenteric vein; LMV: lower mesenteric vein.

Table 3. Distribution of patients according to the associated lesions $(n=17)$.

\begin{tabular}{ccc}
\hline Associated lesions & Number of cases & Percentages \\
\hline Mesenteric infiltration & 08 & $47 \%$ \\
Splenic infarction & 06 & $35 \%$ \\
Renal infarction & 03 & $17.6 \%$ \\
Liver infarction & 01 & $5.9 \%$ \\
Heterogenousliver & 02 & $11.7 \%$ \\
Portal hypertension (High Blood Pressure) & 03 & $17.6 \%$ \\
Intra-abdominalhydro-aerialcollection & 02 & $11.7 \%$ \\
Acute cholecystitis & 01 & $5.9 \%$ \\
Intra-abdominal tumors & 05 & $29 \%$ \\
Chronic nephropathy kidneys & 04 & $23.5 \%$ \\
\hline
\end{tabular}

that is splenic hepatic and renal in 10 cases; mesenteric infiltration in 08 cases, intra abdominal tumor in 05 cases, cholecystitis in 01 case and septic collection in 02 cases.

\subsubsection{CT Diagnostics}

We diagnosed 16 cases of acute mesenteric ischemia and 04 cases of ischemic colitis. According to the scanner-suspected seriousness, 11 cases of infarction were noted. Our 16 cases of acute mesenteric ischemia (AMI) were divided into 12 cases of arterial AMI, 2 cases of venous AMI and 2 cases of mixed AMI. The 12 cases of arterial AMI were due to thrombosis in 07 cases, embolism in 04 cases and low flow in 01 case.

\subsection{Therapeutic and Evolutionary Data}

Ten (10) patients benefitted from an exclusive medical treatment, among them, 5 patients were lost to follow-up. The ten patients operated had peroperative intestinal necrosis. In their evolution 13 patients died. 


\section{Discussion}

Our study had some limitations, especially the small size of our sample; the high number of patients lost to medical treatment who may have underestimated the mortality rate. Also we could not specifically link each CT sign to an intraoperative data. Nevertheless, our results are appreciable and we were able to lead a discussion by comparing them and illustrating them by the data of the literature.

Acute intestinal ischemia is a rare and serious disease [1] [5]. The frequency of 2.2 cases per month is higher than the one noted by Kassaiaa in Morocco ( 0.3 cases per month) [6] and by Sanouin Burkina Faso (0.1 case per month) [7]. Though it is rare, the frequency of mesenteric ischemia is increasing due to the combined fact of the increase of the population at risk and the improvement of diagnosis means, especially the access to angioscan [2] [5]. It is a disease of the elderly subject; the average age in our series is 67.8 years. It was 70 years in the series of Nuzzo et al. in France [2] [8]. Cardiovascular history was often noted in $35 \%$ of patients in our series. [3] [8]. Abdominal pain was the ground for consultation in all cases. A catastrophic abdominal pain contrasting with a normal clinical examination is the standard classic in acute mesenteric ischemia at the early stage. When physical symptoms are found like in half of our series, it means there is intestinal necrosis [9]. The average deadline for the scanner which is 54 hours after the outbreak of the symptoms may justify the bad prognosis of the cases studied. In fact, at the physiopathological level, beyond the twelfth hour after the outbreak of the pain, start the lesions of intestinal necrosis [9] [10] [11].

Compared to the study by Leithmakiin Finland, the clinician suspected the diagnosis in 50\% of cases against 30\%. A good diagnosis can be achieved through an appropriate acquisition protocol and by informing the radiologist of the suspected diagnosis [12] [13]. The lack of parietal heightening, noted in 13 cases over 20 is a quite specific symptom of acute intestinal ischemia (96\% to 100\%) but less sensitive (18\% to 62\%) [4] [12]. The lack of heightening, as well as intestinal distention is significantly associated with peroperative intestinal necrosis [3] [4] [12]. Parietal pneumatosis also makes to suspect transmural necrosis. Cases of benign parietal pneumatosis were described, but when they are associated with aeromesentery, they may be pathognomonic of intestinal necrosis [3] [4] [8]. The parietal thickening noted in 12 cases shows there is a mural oedema, and is most often reversible [2] [4]. It is the same for the severe parietal heigthening noted in 03 cases. In our series, these symptoms of reversible ischemia were often associated with other pejorative symptoms such as the lack of parietal thickening and parietal pneumatosis. The lack of bowel suffering symptoms revealed by the scanner was noted in 02 cases. Generally, in such cases, the patient is sent back home by health workers wrongly reassured by a normal physical examination, biology and scanner [10] [12]. Partial or total UMA was found in 10 cases. This confirms the fact that the cause is often arterial $(75 \%$ in our series). Emile SH et al., over 101 patients, showed that the UMA occlusion is a 
predictive factor of intestinal necrosis [4] [14]. Venous occlusion concerned the portal trunk in 03 cases and UMV in 03 cases. Portal thrombosis is often favoured by the existence of portal high blood pressure, but it may be secondary to the extension of the UMV thrombosis [12] [15] [16]. UMV thrombosis is generally related to an infectious, inflammatory or digestive tumoral disease [4] [15] [17]. The lack of vascular occlusion was noted in 07 cases and often seen in cases of ischemic colitis or low flow arterial occlusion. It should be noted that in arterial occlusions due to embolism, the emboli, when too distal, may not be recognized during the scanner [2] [4] [18]. Thus, several situations may lead to an unknown diagnosis:

- The clinical trap before a poor clinical examination.

- The biological trap before normal and non-specific biological examinations.

- The radiologicaltrap:

$\checkmark$ Before a normal abdominal scanner at the early stage with a lack of digestive suffering, we must make sure a good technique is used (abdominal CT angiography) and search for the vascular obstruction that will lead tothe diagnosis.

$\checkmark$ Before symptoms of digestive suffering without viewed vascular obstruction, we must remember that too distal emboli may not be well seen by the CT angiography, and therefore suggest the diagnosis if the clinical situation is concordant.

$\checkmark$ Finally, be able to repeat an angioscan a few hours later, if there is a clinical suspicion and if the initial scanner is normal.

The death rate of $65 \%$ in our seriesis almost similar to that of Kassaai $60 \%$ and inferior to that of Sanou 100\% [6] [7]. Diagnosis is often late and the outcome fatal [7] [15] [19] [20]. When the patient survives he is often develops the short bowel syndrome [9] [10] [12].

\section{Conclusion}

Acute intestinal ischemia is a rare but serious disease. Multi-detector CT scan has changed vascular explorations. Nowadays abdominal CT angiography is the gold standard to confirm a diagnosis. A good diagnosis requires a high level of clinical suspicion clearly mentioned in the request addressed to the radiologist. Improved prognosis starts with early diagnosis.

\section{Acknowledgements}

The authors would like to thank professor Mizouni, the head of department of radiology of Rabta Hospital of Tunis for allowing them to collect these data, in order to write this article. We also thank the whole staff of the radiology department of RABTA Hospital, Tunis.

\section{Ethics and Consent}

We got the authorization from the Hospital's director, as well as all the various 
heads of the units concerned. We also got the informed consent of the patients or their dependents. Anonymity was respected for all the patients.

\section{Funding Information}

The study did not receive any funding.

\section{Author Contribution}

All authors contributed to write the manuscript. All authors read and approved the final manuscript.

\section{Conflicts of Interest}

The authors have no competing interests to declare.

\section{References}

[1] Vilgrain,V. and Regent, D. (2010) Abdominal Imaging. Lavoisier, Paris, 1055 p.

[2] Otal, P. (2016) Mesenteric Ischemia: Radiological Semiological Elements. JFR 2008, Coming from the Everyday Life of JFR'08, Tuesday 28th October. http://www.sfrnet.org/formation/mediatheque/Textes/02\%20-\%20Cardiovasculaire \%20diagnostique\%20et\%20interventionnel/article.phtml?id=rc\%2Forg\%2Fsfrnet $\% 2$ Fhtm\%2FArticle\%2F2009\%2Fhtm-20090410-131253-667

[3] Nuzzo, A., Joly, F., Ronot, M., et al. (2013) Prognostic Factors of Intestinal Resection and Long-Term Survival after Acute Mesenteric Ischemia: A Retrospective Observational Cohort Study of 164 Survivors. Réanimation, 23, S41-S42.

[4] Delhom, E, Aufort, S., Shrembi, V., Lonjon, J., Bruel, J.M. and Gallix, B. (2009) Acute Ischemia of the Mesentery: Contributions of Imaging in Sections. Radiology Journal, 92, 1060-1071. https://doi.org/10.1016/j.jradio.2011.03.029

[5] Bennaceur, R., Boughanmi, N. and Ben Amara, F. (2009) The Interest of Multi-Slice $\mathrm{CT}$ in the Exploration of Mesenteric Ischemia. Medical Imaging Department, Charles Nicolle Hospital, Tunis,

http://www.pe.sfrnet.org/Data/.../pdf/2009/1/19b12bd7-6d04-458e-bfb5-97537baad 594.pdf

[6] Kassaiaa, F.Z. (2010) Mesenteric Infarction about 19 Cases. Human Medicine: Marrakech, $154 \mathrm{p}$.

[7] Entero S.S. (2016) Mesenteric Infarction: Epidemiological, Clinical, Paraclinical, Therapeutic and Prognostics Aspects: About 7 Cases at CHU YO. Doctoral Thesis No. 351, UFR/SDS of the University of Ouagadougou, Burkina Faso, Medicine Section, $42 \mathrm{p}$.

[8] Barral, M., Boudiaf, M., Dohan, A., et al. (2016) CT Aspects of Acute Colitis in Adults. Journal of Diagnostic and Interventional Radiology, 96, 6-22. https://doi.org/10.1016/j.jradio.2014.03.013

[9] Bala, M., Kashuk, J., Moore, E.E., et al. (2017) Acute Mesenteric Ischemia: Guideline of the World Society of Emergency Surgery. World Journal of Emergency Surgery, 12, 38. https://doi.org/10.1186/s13017-017-0150-5

[10] Nuzzo, A. and Corcos, O. (2013) Acute Mesenteric Ischemia in 2013: A Multimodal and Multidisciplinary Approach Coordinated by the Gastroenterologist. Mini-Journal of Hepatogastro and Digestive Oncology, 20, 461. 
[11] Milone, M., Dario Di Minno, M.N., Musella, M., et al. (2013) Computed Tomography Findings of Pneumatosis and Portomesenteric venous Gas in Acute Bowel Ischemia. World Journal of Gastroenterology, 19, 6579-6584. https://doi.org/10.3748/wjg.v19.i39.6579

[12] Nuzzo, A. and Corcos, O. (2017) Management of Mesenteric Ischemia in the Era of Intestinal Vascular Emergency Structures. Revue De Medecine Interne, 38, 592-602. https://doi.org/10.1016/j.revmed.2017.01.018

[13] Lehtimäki, T.T., Kärkkäinen, J.M., Saari, P., Manninen, H., Paajanen, H. and Vanninen, R. (2015) Detecting Acute Mesenteric Ischemia in CT of Acute Abdomen Is Dependent on Clinical Suspicion: Review of 95 Patients. European Journal of Radiology, 84, 2444-2453. https://doi.org/10.1016/j.ejrad.2015.09.006

[14] Emile, S.H. (2018) Predictives Factors for Intestinal Transmural Necrosis in Patients with Acute Mesenteric Ischemia. World Journal of Surgery, 42, 2364-2372. https://doi.org/10.1007/s00268-018-4503-3

[15] Rafael, D., Alban, D., Pierre, S. and Sabine, S. (2009) Venous Mesenteric Thrombosis, Tomodensitometric Semiology According to the Underlying Pathology. Electronic French Day Radiology Poster 2009.

http://www.pe.sfrnet.org/Data/.../pdf/2009/1/fc4d634e-2f88-4dc0-87dd-37eaf6c4464 d.pdf

[16] Benjilali, L. and Essaadouni, L. (2016) Splanchnic Venous Thromboses: A Monocentric Study of 31 Cases. Journal of Vascular Diseases, 41, 26-35.

[17] Rachid, E.B., Abdennasser, E.K., Mohamed, L., et al. (2013) Superior Mesenteric Venous Thrombosis Complicating Unrecognized Appendicitis. Pan African Medical Journal, 14, 12.

[18] Bourcier, S., Goudard, G., Oudjit, A., et al. (2013) Epidemiological Study of Mesenteric Ischemia Acquired in Intensive Care. Réanimation, 23, S41.

[19] Plumereau, F., Mucci, S., Lenaoures, P., Finel, J.B. and Hamy, A. (2015) Acute Mesenteric Ischemia of Arterial Etiology: Benefit of Early Revascularization. Journal of Visceral Surgery, 1, 17-22. https://doi.org/10.1016/j.jviscsurg.2014.11.001

[20] Ka, O., Sanou, A., Dieng, M., et al. (2009) Acute Mesenteric Ischemia: Diagnostic and Therapeutic Aspects of 13 Cases. African Journal Digestive Surgery, 9, 872-875. 